\title{
Thermalized collisional pre-sheath detected in dense plasma with coherent and incoherent Thomson scattering
}

\author{
J. van den Berg-Stolp, H.J. van der Meiden, I.G.J. Classen, J.W.M. \\ Vernimmen, Y. Li, J. Scholten, S. Brons, G.J. van Rooij \\ DIFFER (Dutch Institute for Fundamental Energy Research), De Zaale 20, 5612 AJ \\ Eindhoven, The Netherlands \\ E-mail: $j$.vandenberg@differ.nl
}

\begin{abstract}
In the direct vicinity of plasma-facing surfaces, the incident plasma particles interact with surface-recombined neutrals. Remarkably high near-surface plasma pressure losses were observed in the high-flux linear plasma generator Magnum-PSI. Combining the incoherent and coherent Thomson scattering diagnostics, we directly measured particle, momentum and energy fluxes down to $3 \mathrm{~mm}$ from the plasma target surface. At the surface, the particle and total heat flux were also measured, using respectively an in-target Langmuir probe and thermographic methods.

The near-surface momentum and energy losses scale with density, and amount to at least $50 \%$ and $20 \%$, respectively, at $n_{e}=8 \cdot 10^{20} \mathrm{~m}^{-3}$. These losses are attributed to the efficient exchange of charge, momentum and energy between incident plasma and surface-recombined neutrals. In low-temperature plasmas with sufficient density, incident particles go through several cycles of interaction and surface deposition before leaving the plasma, thereby providing an effective alternative dissipation channel to the incident plasma.

Parallel plasma parameter profiles exhibit a transition with increasing plasma density. In lowdensity conditions, the plasma temperature is constant and near-surface ion acceleration is observed, attributed to the ambipolar electric field. Conversely, deceleration and plasma cooling are observed in dense conditions. These results are explained by the combined effect of ionneutral friction and electron-ion thermal equilibration in the so-called thermalized collisional pre-sheath. The energy available for ambipolar acceleration is thus reduced, as well as the upstream flow velocity.

In the ITER divertor, enhanced near-surface p-n interaction is expected as well, given the overlap in plasma conditions. Including these effects in finite-element scrape-off layer models requires a near-surface resolution smaller than the neutral mean free path. This amounts to $1 \mathrm{~mm}$ in Magnum-PSI, and possibly an order of magnitude smaller in ITER.
\end{abstract}

\section{Introduction}

The interaction between surface-recombined neutrals and incident plasma in tokamak divertors is an essential aspect of Scrape-Off Layer (SOL) physics [1,2]. Elastic as well as inelastic collisions facilitate mutual exchange of charge, momentum and energy. The net effect of plasma-neutral (p-n) interaction on the SOL plasma depends generally on three plasma properties: temperature, density, and residence time of neutrals in the interaction region. The plasma temperature determines which interactions are present, be it ionization, elastic collisions or recombination. 
The plasma and neutral densities $\left(n_{e}\right.$ and $\left.n_{n}\right)$ determine the reaction rate of those interactions. Finally, plasma dimensions and geometry determine the residence time of neutrals in the SOL, and therewith the total interaction potential, as has been shown in (modeling) experiments with closed divertor concepts [3], as well as target closure experiments on linear devices [4]. In a process called divertor detachment, p-n interaction gives rise to momentum and energy losses in the SOL, thus lowering the particle and heat loads on the divertor target surface $[5,6]$.

The region in direct vicinity of the target surface is of particular interest regarding p-n interaction, because it encompasses an intense source of neutrals: surface recombination. This near-surface region receives an inflow of ions towards the sheath, matched by outwards diffusion of surface-recombined neutrals. Ions and neutrals interact, and for sufficiently high interaction rates, their velocity distributions are mutually influenced. That is, the ion and neutral populations are (partially) coupled. This process is therefore called near-surface plasma-neutral coupling (shortly p-n coupling). Neutral particles are not confined in the magnetic field, and dissipate energy from the near-surface system through radiation, diffusion, or surface deposition. The latter process leads to a cycle of exchange and deposition. Each incident particle can experience several cycles, before being either lost or ionized. Near-surface p-n coupling thus opens up an efficient dissipation channel, resulting in significant and highly localized losses of plasma pressure and power flux.

The flux of charged particles onto the wall is governed by the plasma sheath. An electrically floating wall is charged negatively by the highly mobile electrons, resulting in an electric potential drop near the surface. The corresponding ambipolar electric field accelerates the ions towards the surface, reaching the velocity of sound at the sheath edge. Ion-neutral friction and electron cooling in the near-surface region can interfere with this process, affecting the sheathtransmitted PFC loads, but also the conditions upstream.

In this study, these effects of p-n coupling are experimentally assessed in the linear plasma generator Magnum-PSI. These experiments show that near-surface p-n coupling indeed arises in dense plasmas, relevant to the ITER divertor in high-recycling or detached scenarios, and that it causes significant pressure and power losses, in a region that extends less than $10 \mathrm{~mm}$ from the surface.

Previous studies have already found various indications of $\mathrm{p}-\mathrm{n}$ coupling. Modelling studies of the SOL showed strong correlations between the electron temperature $T_{e, t}$ and neutral density near the divertor target, but also between the target parameters and global SOL losses [7]. A more comprehensive study showed that $T_{e, t}$ is a good indicator for divertor detachment in general [5]. One may notice that the target parameters are a product of upstream SOL processes and inputs from the core plasma, but the opposite relation holds as well: the near-surface region prescribes a boundary condition to the plasma upstream. In experimental studies on high-density linear plasmas, estimations of particle and heat flux, using in-plasma incoherent Thomson scattering (TS) and assuming a frictionless and isothermal plasma, have been repeatedly found to be larger than direct measurements at the plasma-facing target surface [8,9]. Moreover, a reduction of electron temperature up to $30 \%$ has been observed, within $10 \mathrm{~mm}$ from the target surface in Magnum-PSI [10].

Experimental quantification of the p-n coupling effects, however, has been limited by the absence of an accurate and direct diagnostic method for the parallel plasma velocity $v_{p}$.

With the commissioning of the coherent Thomson scattering (CTS) diagnostic, such a method has now become available on Magnum-PSI. Using this diagnostic, we measured the ion temperature $T_{i}$ and parallel plasma velocity in the plasma beam center. In combination with incoherent TS, this enabled the first direct, local measurements of the plasma fluxes in Magnum-PSI. Both diagnostics were used down to $3 \mathrm{~mm}$ from the target surface, to produce a series of parallel parameter profiles under various plasma conditions. The particle and total heat flux were also 
measured on the target surface, using a newly developed in-target Langmuir probe, and thermography diagnostics, respectively. The setup and methods of these experiments are described in section 2. Readers interested in the plasma physics results can proceed to section 3 and onwards. In section 4, we show that p-n coupling is present in Magnum-PSI, assess its effects on plasma flow, and estimate the near-surface loss factors for the particle, momentum and energy flux. The interaction processes of p-n coupling are examined in section 5. Finally, the implications of these findings, in particular for the ITER divertor and related SOL modeling, are discussed in section 6 .

\section{Experimental work on Magnum-PSI}

This section describes the setups and methods used to perform the experimental part of this study. The measurement results and interpretation can be found in sections 3 and 4 . In the linear plasma generator Magnum-PSI, a cascaded arc plasma source generates a high-density thermal plasma [11]. The plasma is confined and led to a target by a superconducting magnet (Magnetic field $B<2.5 T$ ). A three-staged differential pumping scheme ensures a low background pressure in the target chamber [12], while maintaining electron densities $n_{e} \lesssim 1.5 \cdot 10^{21}$ in steady state operation. Magnum-PSI is used for various research purposes, including PFC testing [13, 14], PFC diagnostics development, detachment physics studies [15, 16], exploration of novel PMI regimes $[17,18]$ and more.

To investigate near-surface p-n coupling and its effects on the wall loads, the TS and CTS diagnostics (section 2.1) were complemented by two surface diagnostics: an in-target Langmuir probe and a thermographic heat flux measurement (presented in sections 2.2.1 and 2.2.2, respectively). The measurement results are presented in sections 3 and 4.3 for the in-plasma and on-surface measurements, respectively. An overview of the main diagnostics in this research is shown in figure 1.

\subsection{CTS and TS plasma flux measurements in the near-surface region}

Active laser diagnostics are a valuable tool for non-invasive measurement of plasma parameters. This section describes the diagnostics setup used to perform direct plasma flux measurements on Magnum-PSI. The incoherent Thomson Scattering (TS) diagnostic employs a pulsed Nd-YAG laser at $532 \mathrm{~nm}$ to measure electron density $n_{e}$ and temperature $T_{e}$ and is routinely used on Magnum-PSI [19]. The coherent Thomson scattering (CTS) diagnostic is relatively new. It has been developed for use in high-density, low-temperature plasmas, and performed its first measurements on Magnum-PSI's predecessor Pilot-PSI [20]. The CTS system consists of a seeded Nd-YAG laser at $\lambda_{0}=1064 \mathrm{~nm}$, in conjunction with a high-resolution Echelle grating spectrometer featuring a gated short wave infrared camera (Intevac LIVAR M506). This diagnostic system has now been integrated into the TS laser beam line of Magnum-PSI. In these experiments, the laser-surface measurement distance $d$ was minimized. As will become clear in this section, the limiting factor to $d$ is laser stray light. The CTS theory, setup and data analysis are presented in the subsections below.

2.1.1. CTS theory Thomson Scattering (TS) is elastic scattering of electromagnetic waves from electron density fluctuations in ionised gases. The characteristic length scale of the observed fluctuations is given by the scattering wave vector $\boldsymbol{k}=\boldsymbol{k}_{\boldsymbol{s}}-\boldsymbol{k}_{\mathbf{0}}$, with $\boldsymbol{k}_{\boldsymbol{s}}$ and $\boldsymbol{k}_{\mathbf{0}}$ the wave vectors of scattered and incident radiation, respectively. The ratio of this length scale to the Debye length $\lambda_{D e}$, is called the scattering parameter $\alpha=1 /|\boldsymbol{k}| \lambda_{D e}$. For $\alpha \ll 1$, TS observes dominantly scattering from the random motion of the electrons. The resulting incoherent TS spectrum is a direct measure of the electron velocity distribution. For $\alpha \gtrsim 1$, the TS spectrum 

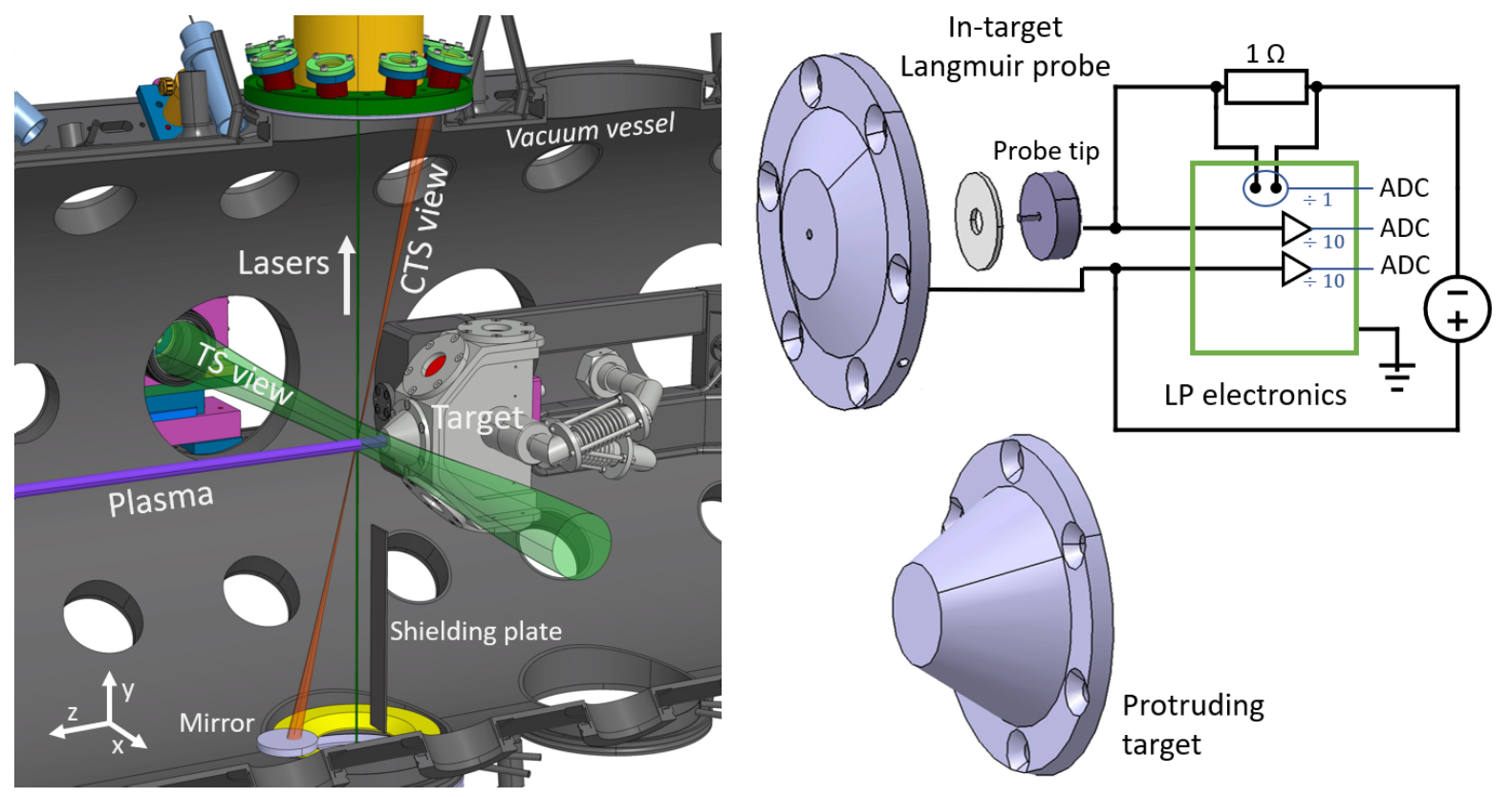

Figure 1. Schematic overview of active laser scattering diagnostics in the vacuum vessel target chamber of Magnum-PSI and plasma targets used. The plasma beam (along the z-axis) is shown in purple. During the CTS and surface heat flux measurements, the protruding target was used. It is conically shaped, to divert stray light in the laser direction away from the viewing optics. The in-target Langmuir probe is depicted as connected, using coaxial cables, to the electronics for power supply and data aqcuisition. The green rectangle represents the aqcuisition electronics, with signal amplifications given in blue. Both targets are made from a titanium-molybdenum-zirconium alloy, TZM.

reflects collective electron motion due to Debye shielding. In this regime of Coherent Thomson Scattering (CTS), the spectrum is sensitive to ion dynamics, and thus can be used to determine $T_{i}$ and $v_{i}$.

Following the Salpeter approximation [21], the CTS spectrum can be represented by two spectral features: one for ion and one for electron shielding. The CTS diagnostic at Magnum-PSI was designed to measure the narrower ion feature. Its shape is given by the so-called form factor $[22]:$

$$
S_{i}(k, \omega) d \omega=\frac{Z}{\sqrt{\pi}}\left(\frac{\alpha^{2}}{1+\alpha^{2}}\right)^{2} \frac{\exp \left(-x_{i}^{2}\right)}{\left(1+\beta^{2} R w\left(x_{i}\right)\right)^{2}+\left(\beta^{2} I w\left(x_{i}\right)\right)^{2}} d x_{i},
$$

where $\omega$ is the frequency of scattered radiation, $x_{i}=\Delta \omega / k v_{t h}$ the normalized wavelength (with $v_{t h}=\sqrt{2 k_{B} T_{i} / m_{i}}$ the average thermal speed for ions). The functions $R w\left(x_{i}\right)$ and $\operatorname{Iw}\left(x_{i}\right)$ are respectively the real and imaginary terms of the plasma dispersion function [21]. Finally, $\beta=\sqrt{Z\left(\frac{\alpha^{2}}{1+\alpha^{2}}\right) \frac{T_{e}}{T_{i}}}$, and $Z$ the effective ion charge, here assumed to equal unity.

It can be seen that $T_{i}$ affects the shape of the ion feature, where the ion velocity distribution is assumed to be Maxwellian. Plasma flow causes a shift of the distribution, that is translated to $S_{i}(k, \omega)$ through projection on $\mathbf{k}$. It is noted that strictly speaking, $T_{i}$ is also measured along $\mathbf{k}$, which main component is parallel to $\mathbf{B}$. 
2.1.2. $\boldsymbol{C T S}$ setup The CTS setup has been made such that the CTS scattering volume overlaps with TS in the plasma center. As such, TS and CTS measurements can be performed simultaneously. The lasers and spectrometers are situated in a separate room from the Magnum hall. Both lasers follow the same beam line of $\sim 25 \mathrm{~m}$ towards the vessel, facilitated by a dichroic mirror in the laser room and a dichroic lens with $f=3.3 \mathrm{~m}$ at 532 as well as $1064 \mathrm{~nm}$ to focus the beams.

Since employment on Pilot-PSI, the laser used for CTS has been upgraded from 10 to $20 \mathrm{~Hz}$, doubling the average laser power to $20 \mathrm{~W}$. A second adaptation with respect to the Pilot-PSI setup, is the change of scattering angle from $30^{\circ}$ to $\theta=13^{\circ}$. This increases the scattering parameter $\alpha$ and CTS signal strength, but reduces the drift-induced Doppler shift of the ion feature, as described in the following subsection 2.1.3.

Another effect of lower $\theta$ is the reduced deflection required for laser light to end up in the viewing system. Given the tiny (C)TS scattering cross section, stray light reduction is an important aspect of TS diagnostic setups. This is even more stringent in the current CTS setup, where not only the stray light signal and ion feature overlap, but the typical spectral width of the latter ( $45 \mathrm{pm}$ at $T_{i} \approx 2 \mathrm{eV}$ ) is only a few times the instrument function of the spectrometer $(12 \mathrm{pm}$ ). There are two main sources of stray light in the vessel: the injection window, located $2.5 \mathrm{~m}$ below the plasma, and the laser dump, located $2.5 \mathrm{~m}$ above the plasma. Stray light from the injection window is mitigated by two laser apertures in the injection tube. A vertical masking plate was placed in the vessel, blocking these apertures from direct view. To minimize stray light from the ejection window, a new laser dump was installed, designed to catch both transmission and reflection from the vacuum exit Brewster window. Finally, a mirror was placed on the bottom of the vessel in the viewing cone, effectively moving the viewing dump towards a faraway section of dark vessel wall.

To perform a CTS measurement, three series are collected of 10 to 20 frames each: A stray light reference without plasma, a wavelength reference and the CTS measurement itself. Before any further data analysis, the averaged stray light frame is subtracted from the CTS measurement. However, variations of signal strength were observed between frames. The residual stray light signal was incorporated in the data analysis procedure, which is described below.

2.1.3. CTS data analysis This section describes the data analysis for estimation of $T_{i}$ and $v_{z}$ from a given CTS spectrum. At the basis of this analysis is the model of the ion feature (equation 1 ), in principle a function of $\lambda, T_{i}, T_{e}, n_{e}$ and $\theta$. The scattering angle is fixed during alignment of the viewing system, with some uncertainty: $\theta=13 \pm 0.5^{\circ}$. Both $T_{e}$ and $n_{e}$ are measured through incoherent TS, and thus are known with some uncertainty. The axial plasma velocity can be inferred from the Doppler shift $\Delta \lambda$ of the ion feature with respect to the laser reference. The laser reference signal was found to exhibit two instabilities: a shot-to-shot intensity fluctuation of $\sim 10 \%$ and a random drift in the wavelength direction, within a range of $0.3 \mathrm{pm}$ and a time scale comparable to the measurement duration. This wavelength drift is transmitted to the ion feature and can be interpreted as a shift of $\lambda_{0}: \Delta \lambda_{0}$, with uncertainty $0.3 \mathrm{pm}$. The intensity fluctuation has two effects: firstly an uncertainty in the amplitude of the ion feature, $A_{C T S}$. Secondly, after stray subtraction from the raw measurement, some stray signal can remain. This stray light was included in the model as a nuisance parameter $A_{\text {stray }}$. Finally, the spectrometer instrument function is included in the model through a digital convolution of the theoretical CTS spectrum with a normalized stray light signal $S_{s}$. The complete CTS model is then given by:

$$
M_{C T S}\left(\lambda_{i}\right)=A_{C T S} S_{s} * S_{i}\left(\lambda_{s}, T_{i}, T_{e}, n_{e}, \theta\right)+A_{s} S_{s}\left(\lambda_{i}-\Delta \lambda_{0}\right)+A_{0},
$$

with $\lambda_{s}=\lambda_{i}-\left(\Delta \lambda-\Delta \lambda_{0}\right)$. Although only $T_{i}$ and $\Delta \lambda$ are of interest, the model has nine independent input parameters. Given the correlations between some of these, mainly $T_{i}$ and 


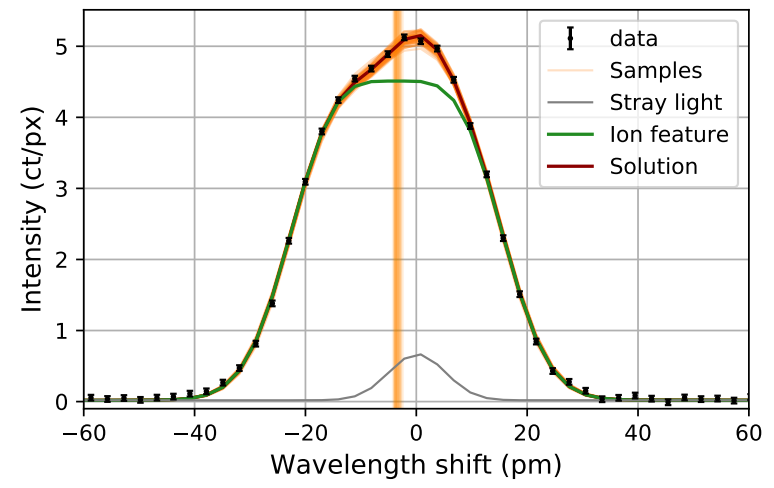

Figure 2. A net CTS spectrum as measured on Magnum-PSI, at $B=1.2 T, H_{2}$ gas flow $7 \mathrm{slm}$, source current $180 \mathrm{~A}, 3 \mathrm{~mm}$ from the target surface. In 31 frames, 806 laser pulses were collected, from which the average of 27 stray light frames was subtracted. The orange vertical lines indicate $\Delta \lambda$ for the samples shown. The outputs are $T_{i}=1.63_{+0.11}^{+0.12}$ and $\Delta \lambda=-3.53_{+0.31}^{+0.31}$.

$\theta$, and their significant uncertainties compared to the model sensitivity, we decided to compare model and data through a Bayesian inference. A previous instance of Bayesian CTS data analysis was implemented on the stellarator Wendelstein-7X [23]. The goal of a Bayesian inference is to obtain the probability distribution for the set of input parameters $\Theta$ given the data $Y=y_{i}$ :

$$
P(\Theta \mid Y)=\frac{P(Y \mid \Theta) P(\Theta)}{P(Y)},
$$

with $P(\Theta)$ the prior probability distribution, $P(Y)$ a normalization that can be discarded, and $P(Y \mid \Theta) P(\Theta)$ the likelihood function. The latter is considered as a Gaussian distribution around the model output, and written in logarithmic form:

$$
\ln P(Y \mid \Lambda, \Theta)=-\frac{1}{2} \sum_{N}\left[\frac{\left(y_{i}-M\left(\lambda_{i}, \Theta\right)\right)^{2}}{\sigma_{y_{i}}^{2}}+\ln \left(2 \pi \sigma_{y_{i}}\right)\right] .
$$

The Gaussian uncertainty is a sum of readout noise and Poisson-distributed shot noise: $\sigma_{y_{i}}=\sigma_{\text {readout }}+\sigma_{\text {shot }, i}$. These are given by data noise outside reach of the ion feature, and by the model outputs as $\sqrt{y_{i}}$, respectively. The posterior distribution is obtained through sampling from the prior distribution. The Monte Carlo Markov Chains (MCMC) algorithm was used for this purpose, as implemented in the EMCEE code [24]. Uniform prior distributions were used some parameters: $T_{i}:[0.1,20] \mathrm{eV}, \Delta \lambda:[-30,30] \mathrm{pm}, A_{C T S}:[0.3, \max (Y)] c t$, and $A_{s}:[-0.9 \max (Y), 0.9 \max (Y)]$ ct. Gaussian distributions for others: $\theta=13 \pm 0.5^{\circ}$, $\Delta \lambda_{0}=0 \pm 0.3 \mathrm{pm}, A_{0}=0 \pm 0.1 \mathrm{ct}$, and $n_{e}$ and $T_{e}$ obtained from incoherent TS with Gaussian uncertainties. In the MCMC algorithm, 32 walkers were set loose, and convergence defined as the chain length that exceeds 60 times the maximum autocorrelation in the samples.

For further processing of the results, the median values and standard deviation of $T_{i}$ and $\Delta \lambda$ were used. However, for direct plots of these parameters, the $16^{\text {th }}$ and $84^{\text {th }}$ quantiles are used as uncertainties in the remainder of this article. An exemplary CTS spectrum with several samples, as well as the median model output, is shown in figure 2 .

From Doppler shift to velocity As stated above, the axial plasma velocity is derived from the Doppler shift $\Delta \lambda$. However, there are two aspects that need to be taken into account 
here. Firstly, measurement $\Delta \lambda$ follows from a projection of $\mathbf{v}$ along the scattering vector $\mathbf{k}$, i.e. $\Delta \omega=\mathbf{k} \cdot \mathbf{v}$. Secondly, next to the axial velocity component $v_{z} \| B, \mathbf{v}$ can contain a plasma rotation component $v_{y}$ if the laser is slightly off-center. The frequency shift is then $\Delta \omega=\mathbf{k} \cdot \mathbf{v}=\mathbf{k v} \cos (\theta / \mathbf{2}-\phi)$, with $\phi=\tan \left(v_{y} / v_{z}\right)$. In combination with $k=2 k_{0} \sin (\theta / 2)$ and the general wave property $\Delta \omega=2 \pi \frac{c_{0}}{\lambda^{2}} \Delta \lambda$ with $c_{0}$ the speed of light, we find the relation:

$$
\frac{\Delta \lambda}{\lambda_{0}}=\frac{v}{c_{0}} \cdot 2 \sin (\theta / 2) \cos (\theta / 2-\phi)
$$

Solving for $v_{z}$, we obtain:

$$
\frac{v_{z}+v_{y} \tan (\theta / 2)}{c_{0}}=\frac{\Delta \lambda}{\lambda_{0}} \frac{1}{\sin (\theta)}
$$

The uncertainties in $v_{z}$ are obtained by error propagation of the partial uncertainties: $u_{v_{y}}=$ $3 \mathrm{~km} / \mathrm{s}$, and $u_{\Delta \lambda}$ as well as $u_{\theta}$ given by the Bayesian analysis.

\subsection{Flux measurements on the target surface}

This section discusses the diagnostics used to assess the effects of p-n coupling on the PFC loads. An in-target Langmuir probe was developed to estimate the surface particle flux $\Gamma$ in the plasma center, as described in section 2.2.1. The total heat flux in the plasma center was measured using a combination of thermographic diagnostics, as presented in section 2.2.2.

2.2.1. Flush-mounted Langmuir probe Langmuir probes are widely used to measure various plasma properties [25], and have also previously been employed in Magnum-PSI [26, 27]. In this research, we used a flush-mounted Langmuir Probe (LP) to estimate the plasma flux in the target center. The surface particle flux is given by

$$
\Gamma_{L P}=\frac{I_{s a t}}{e A_{e f f}}
$$

with $I_{s a t}$ the ion saturation current, $A_{\text {eff }}$ the effective collection area for ions, and $e$ the electron charge. A new probe-in-target system was designed, consisting of a titanium-molybdenumzirconium alloy $(\mathrm{TZM})$ probe pin (plasma-facing surface diameter $1 \mathrm{~mm}$ ) that is clamped onto the multitarget holder by a TZM clamping ring. The probe is water cooled and, during this experiment, withstood heat fluxes up to $20 \mathrm{MW} / \mathrm{m}^{2}$ for several tens of seconds. The probe is electrically isolated from the clamping ring by a $1 \mathrm{~m}$-thick boron nitride plate. A boron nitride coating electrically isolates the probe from the backplate. The probe is connected to the power supply wiring by means of a ceramic-isolated metal screw. The probe pin is facing the plasma in a $2 \mathrm{~mm}$-diameter hole in the clamping ring and is mounted flush to the surface.

Due to radial electric fields from the probe under bias in combination with a finite ion Larmor radius $(0.16 \mathrm{~mm}$ at $1.8 \mathrm{eV}, 1.2 \mathrm{~T})$, the effective collection area $A_{e f f}$ exceeds its front surface area [26]. In this work, we assumed $A_{e f f}=12.6 \mathrm{~mm}^{2}$, equal to the surface of the clamping ring hole. The characteristic I-V curve for a given machine setting is measured by varying the probe potential $V_{p}$ during exposure and measuring the corresponding probe current $I_{p}$. Given that the probe measurements require a different target, they were performed separately from the plasma flux measurements, at equal machine settings. The probe potential is controlled by a bipolar power supply with $I_{\max }=4 \mathrm{~A}$. The probe potential follows a triangular waveform at $100 \mathrm{~Hz}$, between $-50<V_{p}<10 \mathrm{~V}$, generated by a function generator. An isolation amplifier passes this signal to the power supply. 
The probe potential is measured with respect to ground over a $1: 11$ voltage divider. The probe current is determined by measuring the voltage over a $1 \mathrm{Ohm}$-resistor in series with the power supply. For reference, the target holder potential, $V_{t a r}$, was measured. These signals are fed to an 8-channel NI PXI-5105 analog to digital converter at $1 \mathrm{MHz}$. For each machine setting in the experiment, ten seconds of signal were collected, during a TS measurement.

LP data analysis To determine the ion saturation current, we consider the probe surface small compared to the total plasma-wetted area, and employ the single probe LP characteristics. The collected signals are split in five sections. Each section is time-averaged by considering its I-V curve: $I_{p}\left(V_{b}\right)$, with $V_{b}=V_{p}-V_{\text {tar }}$ the relative bias potential. Following the Bohm criterion, ion collection does not depend on bias, while the electrons do not reach the surface if it is sufficiently negatively charged. If voltage sweeping is not feasible, for example due to setup limitations or during transients, $I_{s a t}$ is often assumed to equal $I_{p}$ at a bias that is large compared to $e T_{e}$ [26]. However, radial electric fields emanating from the probe cause increased radial transport and therewith an increase of $A_{\text {eff }}$. Probe-induced radial electric fields are absent at $V_{b}=0$, since the probe then acts as a proper surface element. One can thus estimate $I_{\text {sat }}$ by performing a fit on the negative saturation branch of the I-V curve, and extrapolating to $V_{b}=0$. In (tokamak divertor) conditions of shallow incidence, the Child-Langmuir sheath expansion is frequently used $[28,29]$. In the current setup under perpendicular incidence, this principle is not applicable, and in this work, a simple linear fit was performed in the domain $V_{b}=-50--25 \mathrm{~V}$. The uncertainty in $I_{s a t}$ is given by the standard deviation over the five sections, and propagated into $\Gamma_{L P}$.

2.2.2. Thermographic heat flux estimation The surface heat fluxes were determined by solving the 3D heat equation using the finite element method (FEM) as described in [30]. This model requires several inputs:

- Target geometry and thermal conductivity. The conical protruding target was used for heat flux estimation. The FEM representation of this target is square-shaped but has equal dimensions to the original, and TZM thermal conductivity $104 \mathrm{~W} / \mathrm{mK}$ at $1000^{\circ} \mathrm{C}$.

- Temperatures of target front and back surfaces. The latter was measured directly by a pt100 thermocouple inserted into the target. The peak temperature of the front surface was measured using a FAR Associates ${ }^{\circledR}$ FMPI spectropyrometer with a viewing spot of 4 by $6 \mathrm{~mm}$, aligned to the plasma center. The temperatures were either measured during steady state, or obtained from an exponential fit of the heating curves during exposure.

- The heat flux profile was assumed to be Gaussian. The Full Width at Half Maximum (FWHM) of this profile was determined by analysis of infra red images made during the exposure.

It is noted here that $q_{\text {surf }}$ is strictly not a local measurement: the FEM analysis is sensitive to the surface temperature profile, which is obtained through infra-red imaging. The uncertainties in determining the beam FWHM are incorporated in the uncertainty of $q_{\text {surf }}$. The width of the plasma beam is correlated with $I_{s r c}$ and thus $n_{e}$, and increases from $9 \mathrm{~mm}$ at low density to $13 \mathrm{~mm}$ at $1.4 \cdot 10^{21} \mathrm{~m}^{-3}$.

\subsection{Experimental program}

The main goal of the experimental work was to determine whether near-surface p-n coupling is present in Magnum-PSI, and if so, under which conditions. Plasma density determines the p-n 
interaction intensity, and is thus a key parameter to study. In a series of experiments, $n_{e}$ was varied over two orders of magnitude. This wide operational space was realised by performing three measurement series: a scan of neutral background pressure and a scan of source current during steady state operation, and a measurement series during pulsed operation at various capacitor bank voltages.

The lowest densities and temperatures were realised through gas puffing in the target chamber, which increases the neutral background pressure $p_{n}$. Volumetric recombination and energy losses throughout the length of the beam then result in reduced $n_{e}$ and $T_{e}$ near the target. At a source current of $175 \mathrm{~A}$ and Hydrogen source gas flow $\Gamma_{H 2}=7 \mathrm{slm}$, a background pressure scan was performed, with $p_{n}=0.3-12 \mathrm{~Pa}$, and $n_{e}$ down to $2 \cdot 10^{19} \mathrm{~m}^{-3}$.

In Magnum-PSI, $n_{e}$ is strongly correlated with source current [12]. The second measurement series thus was a scan of source current $I_{s r c}=145-200 \mathrm{~A}$, again at $\Gamma_{H 2}=7 \mathrm{slm}$ but without target chamber gas puffing. During this current scan, $0.25<p_{n}<0.45 \mathrm{~Pa}$, and $2<n_{e} / 10^{20}<14 \mathrm{~m}^{-3}$. For each setting, TS, CTS, LP, and thermography measurements were performed.

Finally, TS and CTS measurements were performed during pulsed operation, which yields higher temperatures and $n_{e}$ up to $3.5 \cdot 10^{21} \mathrm{~m}^{-3}$. A capacitor bank system is attached to the plasma source to produce high-density plasma pulses with a duration of $\sim 1 \mathrm{~ms}$ [31]. TS measurements at various time points in a pulse can be performed by setting a stroboscopic delay between plasma and laser pulse [12]. For CTS, a fixed delay was set, thus accumulating signal over several plasma pulses. By changing this delay as well as the capacitor bank voltage, the plasma parameters were measured in a range of high-density plasma conditions.

All measurements were performed at $B=1.2 T$. During the current and pressure scans, parallel profiles of plasma parameters were made by incrementally moving the target holder along the magnetic field, thereby changing the laser-surface distance $d$. The measurement results are presented in section 3 .

\section{Results}

In this section, the results of the in-plasma and target-surface flux measurements from section 2.3 are presented. We start with the TS and CTS measurements. Figure 3 shows the dimensionless Mach numbers $M \equiv v / c_{s}$ in the parallel direction, as a function of density, during the source current scan and during plasma pulses. It is noted that not only the density, but also $T_{e}$ varies between these measurements: during steady state operation $0.3<T_{e}<2.6 \mathrm{eV}$, while during pulses $8<T_{e}<15 \mathrm{eV}$. As will be discussed in section 5.1, $T_{e}$ does affect the interaction rates, especially for ionization. Therefore, the results in figure 3 are shown separately for steady state and pulsed measurements. In steady state, a strong negative correlation is observed between $M$ and $n_{e}$. This scaling demonstrates the presence of near-surface p-n coupling, as discussed in section 4.1. Axial profiles in the plasma center were made for several settings from the current and pressure scans, for target-laser distances $3 \leq d \leq 50 \mathrm{~mm}$. The results are shown in figures 4 and 5. 


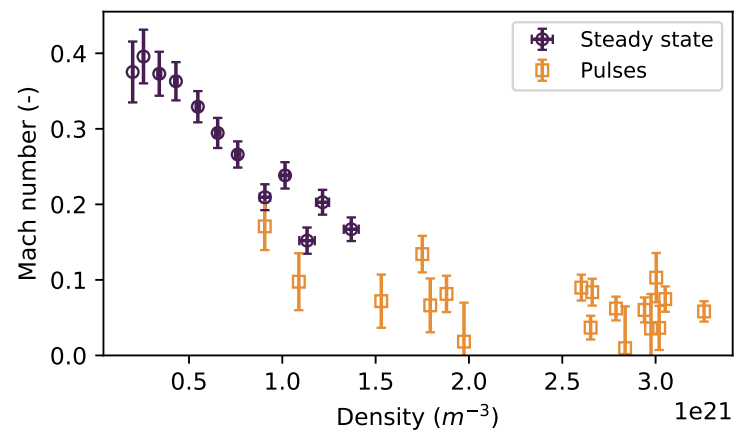

Figure 3. Parallel plasma Mach numbers in Magnum, measured using CTS and TS at the upstream position $d=25 \mathrm{~mm}$ from the target surface, during the steady state source current scan as well as pulsed plasma operation.
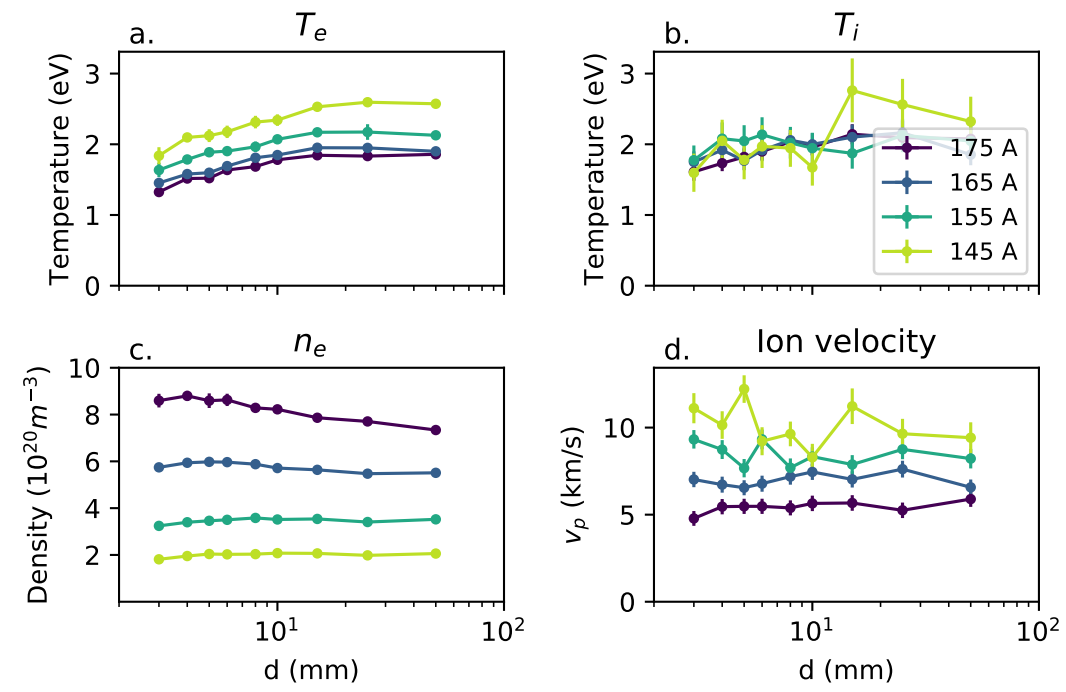

Figure 4. Axial profiles of plasma parameters during a source current scan in MagnumPSI: a. electron temperature, b. ion temperature, c. electron density, and d. parallel ion velocity, obtained using TS and CTS during steady state operation at $B=1.2 \mathrm{~T}, \Gamma_{\mathrm{H} 2}=7 \mathrm{slm}$, $0.25<p_{n}<0.45 P a$, and coloured by source current. 

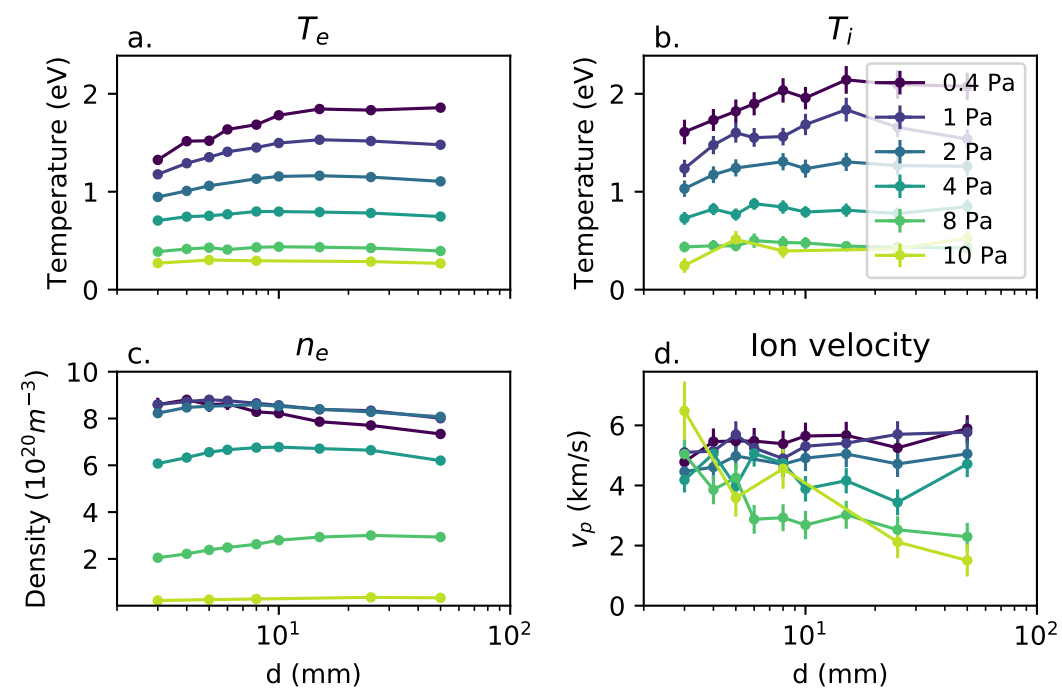

Figure 5. Axial profiles of plasma state parameters during a pressure scan in Magnum-PSI: a. electron temperature, b. ion temperature, c. electron density, and d. parallel ion velocity, coloured by neutral background pressure. Machine settings $B$ and $\Gamma_{H 2}$ equal to those in figure 4, source current $175 \mathrm{~A}$.

In the cases of low plasma pressure (corresponding to high neutral pressure, $p_{n}=8$ and $10 P a$ in figure 5.d), ion acceleration is observed towards the surface, and attributed to pre-sheath ion acceleration through the ambipolar electric field. With increasing plasma pressure, this acceleration disappears, and instead, deceleration is observed. The interpretation of these results is presented in section 4.2 .

The plasma flux measurements are shown in figure 6, as a function of upstream plasma density. Surface measurements are shown in green, and compared to the TS and CTS direct measurements in the plasma, at the near-surface and upstream positions, $d=3$ and $25 \mathrm{~mm}$, respectively. Estimations of momentum and energy flux loss factors, $f_{m o m} \equiv\left(p_{u}^{\text {tot }}-p_{s e}^{\text {tot }}\right) / p_{u}^{\text {tot }}$ and likewise $f_{\text {pow }}$, are shown in subplots e. and f., respectively. The estimations plotted purple were obtained by direct comparison of the measurements at $d=3$ and $25 \mathrm{~mm}$. The momentum loss estimation, plotted red in subplot e., was obtained by solving equation 11 for $f_{\text {mom }}$, and substituting the sheath edge values there with measurements at $d=3 \mathrm{~mm}$. The estimation of $f_{\text {pow }}$, plotted green in subplot f., was obtained by comparing the surface heat flux estimation with the measurements at $d=25 \mathrm{~mm}$. The interpretation of these measurements is presented in section 4.3.

\section{Plasma-neutral coupling in the thermalized collisional pre-sheath}

The TS and CTS measurements presented in the previous section form a characterization of the near-surface plasma in Magnum-PSI, which shows the effects of near-surface p-n coupling. In this section, these effects are discussed. Firstly, near-surface losses affect the plasma flow upstream, as described in subsection 4.1. Secondly, the combination of p-n coupling and electron-ion thermalization in the near-surface region reduces the particle and heat flux into the plasma sheath. The theory of this thermalized collisional pre-sheath and its effects are discussed in subsection 4.2. Finally, the direct plasma flux measurements from TS and CTS are compared measurements and estimations on the target surface in subsection 4.3, yielding estimations of near-surface loss factors for the particle, momentum and energy fluxes in Magnum-PSI. 


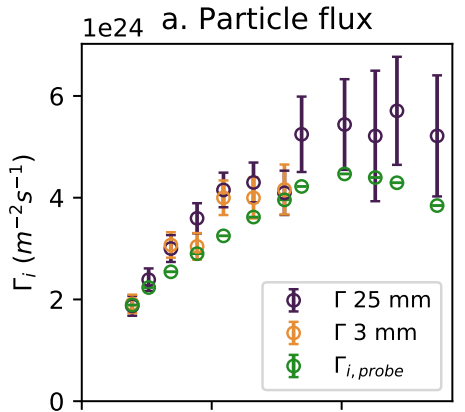

d. Axial velocity

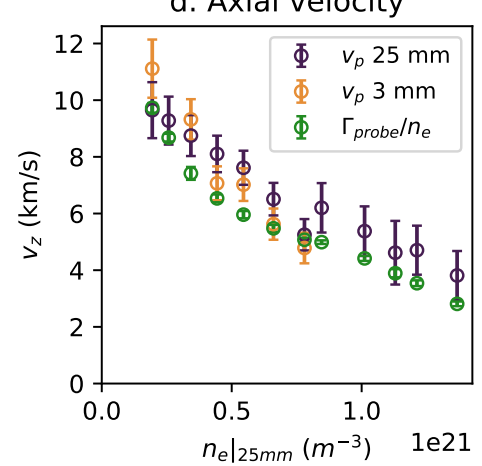

b. Momentum flux

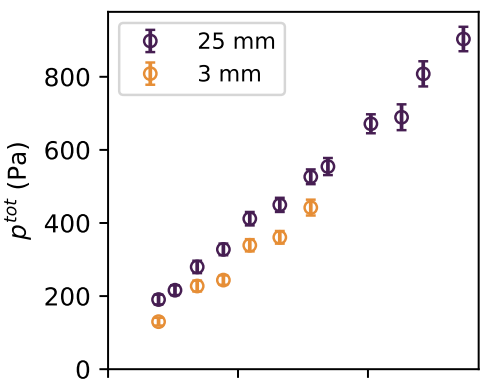

e. Momentum loss

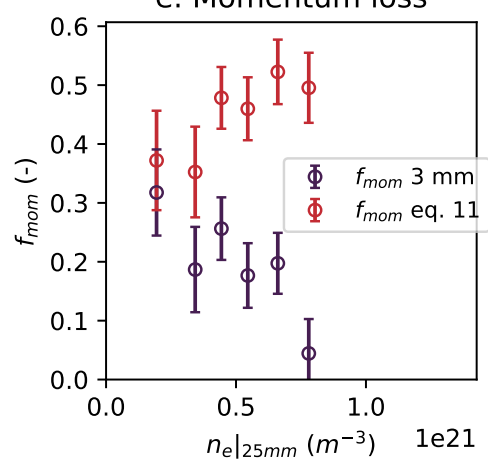

c. Heat flux
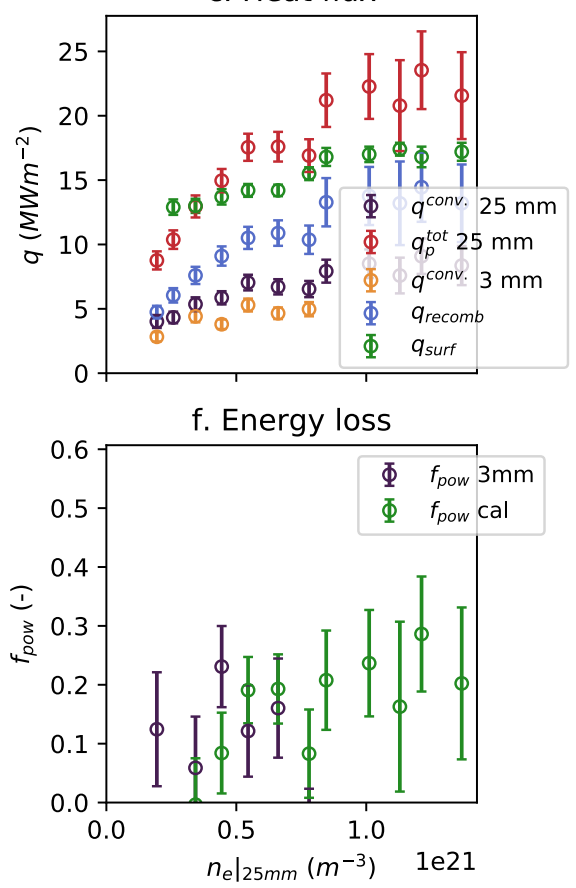

Figure 6. Plasma flux measurements in Magnum-PSI during the source current scan as a function of upstream plasma density. The plasma flux measurements in subplots a, b and c were obtained through equations 8a, 8a, and 8a, respectively, using TS and CTS measurements of $n_{e}$, $T_{e}, T_{i}$, and $v_{i}$ as inputs. The dark purple and orange points correspond measurements at $d=25$ and $3 \mathrm{~mm}$, respectively. The green data is obtained through surface diagnostics. The red data points in plot e. represent an estimation of $f_{m o m}$ through equation 11, using $T_{e}$ at $d=3 \mathrm{~mm}$ for $T_{t}$.

\subsection{Presence of near-surface $p$-n coupling and upstream effects}

Near-surface plasma-neutral coupling entails the transfer of momentum and energy from plasma to neutrals, and causes plasma flux losses. These losses affect the plasma flow upstream of the interaction region. To illustrate this, we consider the system of conservation equations for the fluxes of particles, total momentum and convected energy:

$$
\begin{aligned}
\Gamma & =n v \\
p^{t o t} & =n\left(m_{i} v^{2}+2 e T\right) \\
q^{c o n v} & =n v\left(\frac{1}{2} m_{i} v^{2}+5 e T\right),
\end{aligned}
$$

where $n$ and $T$ are plasma density and temperature, respectively, $m_{i}$ is the ion mass and $e$ the electron charge. Due to low temperatures $\lesssim 10 \mathrm{eV}$ and low impurity concentrations, the effective ion charge can be assumed unity in Magnum-PSI, i.e. $n_{i}=n_{e}=n$. MagnumPSI generates a thermal plasma. Compared to flow time scales, thermal equilibration is fast: $\tau_{e q} \approx\left(2.9 \cdot 10^{-12} n \ln \Lambda T_{e}^{-3 / 2}\right)^{-1} \approx 10^{-10} s$ for Coulomb logarithm $\ln \Lambda=15, n=5 \cdot 10^{20}$ and $T_{e} \approx 2 \mathrm{eV}$ [see 1, eq. 9.52]. Thus, we can assume $T_{e}=T_{i}=T$. This analysis considers 
the convective energy flux. It can be shown that - even for the largest temperature gradients observed - thermal conduction is small compared to convection, and can be omitted here.

To obtain a particle, momentum and energy balance of the near-surface system, the fluxes are evaluated at a position upstream of the interaction region (denoted $u$ ), and at a downstream position: the Debye sheath edge (denoted se). At the sheath edge, the particle flux is given by the Bohm criterion, stating that ions enter the sheath with at least the velocity of sound: $v_{s e} \geq c_{s, s e}$, with $c_{s}=\sqrt{e\left(T_{e}+\chi_{i} T_{i}\right) / m_{i}}$, where $T_{i}$ is ion temperature and $\chi_{i}$ the ion polytropic coefficient. In principle, $\chi_{i}$ is a local parameter, and known to vary considerably in the sheath region [32]. In this study, $\chi_{i} \equiv 1$, setting a lower bound on $c_{s}$. The boundary condition $v_{s e} \geq c_{s, s e}$ is matched in the so-called plasma pre-sheath. In principle, the pre-sheath extends well into the plasma, covering the region of ionization and or cross-field plasma transport. This work considers near-surface plasma-neutral interaction and its effects on pre-sheath processes. In Magnum-PSI, this interaction occurs within ca. $15 \mathrm{~mm}$ from the target surface, as shown in subsection 4.2. The upstream position is thus set at $d_{u}=25 \mathrm{~mm}$.

The particle conservation equation is then given by:

$$
\Gamma_{s e}=n_{s e} c_{s, s e}=n_{u} v_{u}+S_{p}
$$

with $S_{p}$ the integral particle source between the upstream and sheath edge positions. The corresponding momentum conservation equation reads:

$$
n_{u}\left(m_{i} v_{u}^{2}+2 e T_{u}\right)\left(1-f_{m o m}\right)=n_{s e}\left(m_{i} c_{s e}^{2}+2 e T_{s e}\right),
$$

with $f_{m o m} \equiv\left(p_{u}^{t o t}-p_{s e}^{t o t}\right) / p_{u}^{t o t}$ the momentum loss factor. It is noted that at the sheath edge, kinetic pressure equals static pressure, i.e. $m_{i} c_{s, s e}^{2}=2 e T_{s e}$. For $v_{u}<c_{s, s e}$, the pre-sheath entails a conversion of static to kinetic pressure, that is, plasam acceleration. To evaluate the effect of p-n interaction on plasma flow, quations 9 and 10 can be solved for $v_{u}$. In absence of any source terms $\left(S_{p}=0, f_{m o m}=0\right.$ and $\left.T_{s e}=T_{u}\right)$, one obtains the trivial solution: $v_{u}=c_{s, u}$. Therefore, $v_{u}<c_{s, u}$ implies the presence of at least one non-zero source term between $d_{u}$ and the surface. In Magnum-PSI steady state operation, the particle source can be considered small, as is shown in sections 4.2 and 4.3. Combining equation 10 with the boundary condition $\Gamma_{s e}=n_{s e} c_{s, s e}$, one can obtain an alternative expression for the particle flux [5]:

$$
\Gamma_{s e}=\sqrt{\frac{2 e}{m_{i}}} \frac{\left(1-f_{m o m}\right) p_{u}^{t o t}}{4 e \sqrt{T_{s e}}} .
$$

This equation shows that for a given upstream pressure, momentum losses act to reduce the particle flux, while energy losses $\left(T_{s e}<T_{u}\right)$ have the opposite effect.

Figure 3 shows the measured upstream Mach numbers in Magnum-PSI during the current scan and plasma pulses. During the current scan, $M_{u}<0.5$, and a clear correlation is observed between $n_{e}$ and $M_{u}$ : the denser the plasma, the slower it flows. These measurements thus indicate that near-surface plasma-neutral interaction is present in Magnum-PSI, and that it scales with density during steady state operation.

In pulsed operation, the plasma is almost completely stagnant: $M<0.1$. Following equation 11, this implies very strong momentum losses. During pulses, the plasma is in the ionizing regime, with $T_{e}=8-15 \mathrm{eV}$. Particle flux is then not conserved.Nevertheless, strong momentum and energy losses can be expected in this regime, as is explained in section 5.1. Although $M$ is very low in these conditions, it does not show a strong dependence on $n_{e}$. A possible explanation, assuming that plasma equilibration is fast compared to pulse duration, is that if the plasma were to stagnate completely, the surface recombination source would also dry up. In practice, an equilibrium is found between all available processes, leading to a small, but non-zero flow velocity upstream. 


\subsection{Axial profiles show thermalized collisional pre-sheath}

We now study the near-target region in more detail. The axial profiles show various effects of p-n coupling and give insight in the governing processes of the plasma pre-sheath. The main observations in the axial profiles of figures 4 and 5 concern the plasma temperature and plasma velocity.

We first consider the plasma temperature. The ion and electron temperatures are in good agreement with the assumption of thermal equilibration $\left(T_{e}=T_{e}\right)$ in this domain. Furthermore, the temperature is found to decrease towards the surface: during the current scan (fig. 4), the cooling amounts to $25-29 \%$, within $15 \mathrm{~mm}$ from the surface. The axial temperature gradient increases towards the surface, indicating that this is a near-surface effect. Energy lost to the plasma is gained by the neutrals. These measurements therefore demonstrate the presence of near-surface p-n coupling. In the background pressure scan (fig. 5), plasma cooling is observed as well, but the effect decreases with $p_{n}$ and disappears for the highest background pressures 8 and $10 \mathrm{~Pa}$. High background pressures yield high p-n interaction rates over the length of the beam, resulting in reduced plasma pressure in the target region. Low plasma pressure (particularly low $n_{e}$ ) implies low p-n interaction rates, which corresponds well to the observations described above.

Now, the axial velocity profiles of the pressure scan (fig. 5.d) are considered. At high $p_{n}$, plasma acceleration is observed. This acceleration can be attributed to the pre-sheath ambipolar electric field, further discussed below. With increasing plasma pressure (decreasing $p_{n}$ ), plasma acceleration is subdued, and even turned into deceleration. Within errorbars, the reductions in velocity correspond to increases in density, showing that particle flux is conserved in this spatial domain and operation window of Magnum-PSI. This assertion is underlined by LP measurements, as discussed in section 4.3.

Thermalized collisional pre-sheath Both plasma cooling and deceleration depend on density, leading to the distinction of two plasma-surface interaction (PSI) regimes: with and without the presence of p-n coupling. These regimes follow the same plasma physics: ambipolar particle flux towards the wall, regulated by the Debye sheath. Due to the high mobility of electrons, the wall is negatively charged. This charge is provided by plasma electrons, resulting in a positive space charge in the sheath and pre-sheath. This space charge gives rise to the ambipolar electric field, as described by the Poisson equation, see e.g. Stangeby [1, p.72]. The ambipolar field accelerates plasma ions towards the sound velocity. In section 4.1, we considered this pre-sheath acceleration from the viewpoint of pressure conversion (static to kinetic pressure). Here, the perspective of electric potential is considered. The total potential difference required to reach $M_{s e}=1$ is defined as the pre-sheath potential difference, $V_{p r e}$. The sheath potential energy is ultimately provided by the electrons, as is reflected in the electron Boltzmann factor relation:

$$
\frac{n_{s e}}{n_{u}}=e^{\frac{-V_{p r e}}{e T_{e}}} .
$$

In absence of ion-neutral friction, one can find that $V_{\text {pre }}=\ln (2) e T_{e}[1$, eq. 1.53]. In this regime called the collisionless pre-sheath, the ions freely accelerate in the ambipolar field, and the relation between electric potential and ion kinetic energy is fixed. The presence of plasma-neutral interaction introduces the collisional pre-sheath regime: ion-neutral friction increases the electric field required for acceleration and therewith increases $V_{\text {pre }}$. Following equation 12, this leads to a reduction of $M_{u}$, analogous to the results discussed in section 4.1. In the collisional pre-sheath, $V_{\text {pre }}$ depends on the amount of interaction, but holds a fixed relation with the density drop. This effect was quantified by Stangeby [33], for isothermal conditions.

In Magnum-PSI however, near-surface electron cooling is observed. This is attributed to ion- 
neutral energy transfer, i.e. p-n coupling, combined with electron-ion thermal equilibration. In these conditions, the Boltzman factor relation is adapted: $T_{e}$ becomes $T_{e}(z)$. The relation between electric potential and electron density is thus no longer fixed. Pre-sheath deceleration is then possible for sufficiently high temperature gradients. This explains the deceleration observed at high plasma pressures. The ambipolar field is still present, but in the presence of p-n coupling-induced friction and cooling, it is not large enough to keep the acceleration going. Electron cooling simply reduces the energy available for acceleration. Nevertheless, pressure conversion towards sonic flow at the sheath edge boundary is continuous: even with deceleration present, $M$ was never observed to decrease towards the surface. In dense thermal plasmas, near-surface collisions affect the pre-sheath processes, with additional effects due to e-i thermal equilibration. Therefore, we call this the thermalized collisional pre-sheath.

In conclusion, near-surface pre-sheath processes are governed by the momentum and energy losses that arise in p-n coupling: ion-neutral friction causes a reduction of particle flux (for fixed upstream pressure), while plasma cooling reduces $c_{s, s e}$, and therewith the sheath-transmitted plasma flux onto the wall. The interaction intensity is determined in turn by the plasma fluxes. Finally, the near-surface system entails a web of processes, and in itself is only a link in the chain of plasma edge physics.

We briefly examine the shape of the parallel density profiles once more. Assuming $n v=$ const., these are equivalent to the corresponding velocity profiles, albeit with smaller uncertainties. Considering the parallel density profiles in figures 4 and 5 , the acceleration region first emerges close to the surface, and expands into the plasma with the reduction of upstream pressure. This is in good agreement with the ambipolar field being strongest near the surface. Below $d=3 \mathrm{~mm}$, further acceleration is thus still expected, although further cooling is possible as well. Together, these processes satisfy the boundary condition $M_{s e}=1$. The next section of this paper focusses on the effect of p-n coupling on the PFC loads. These loss factors finally determine the fractions of incident energy flowing into the kinetic Debye sheath, or into the neutral gas.

\subsection{Measured loss factors}

The flux losses corresponding to p-n coupling are now quantified. The in-plasma and surface flux measurements during the current scan are shown in figure 6, as a function of density. By comparing measurements at the upstream and near-surface positions, and those on the target surface, the near-surface loss factors are assessed.

Particle flux In line with an almost linearly decreasing velocity (fig. 6.d), the rise of particle flux is reduced with $n_{e}$. The LP shows the same trend, even decreasing for $n_{e}>10^{21} \mathrm{~m}^{-3}$.

In this operation domain, no significant differences are found between the particle fluxes at 25 and $3 \mathrm{~mm}$. The Langmuir probe estimations $\Gamma_{L P}$ are plotted with measured plasma fluxes in plots a. and d. The uncertainty in the effective probe collection area introduces a systematic error. However, by performing a reference measurement after each new setting, the LP sensitivity was confirmed to be constant throughout the current scan. Although there is a small systematic deviation between the probe and CTS velocity estimation, recognized as a constant difference in plot $\mathrm{d}$, their scaling with density is in good agreement. The LP measurements therefore confirm the conservation of particle flux in the near-target region.

Heat flux The total heat flux carried by the plasma is shown in plot c. and estimated as follows:

$$
q_{p}^{t o t}=q^{c o n v}+q^{c o n d}+\Gamma\left(E_{i o n}+E_{H_{2}}\right) .
$$


Here, $q^{\text {cond }}$ is the conduction heat flux, which -given the relatively small temperature gradientscan be assumed to be small compared to convection. The third term represents heat released through surface processes, with $E_{i o n}=13.6 \mathrm{eV}$ for recombination and $E_{H_{2}}=2.2 \mathrm{eV}$ for $H_{2^{-}}$ recombination, and assumes all Hydrogen is recombined at the surface for simplicity. These results are compared with the total surface heat flux on the target surface, $q_{\text {surf }}$, in fig. 6.c and f. While $q_{p}^{\text {tot }}$ follows $\Gamma$ and ranges from 8 up to $23 \mathrm{MW} / \mathrm{m}^{2}, q_{\text {surf }}$ shows a much smaller increase, from 13 to $17 \mathrm{MW} / \mathrm{m}^{2}$. The corresponding power loss factor $f_{\text {pow }}=q_{p}^{\text {tot }} / q_{\text {surf }}$, shown in fig. 6.f, increases significantly with density, especially below $10^{21} \mathrm{~m}^{-3}$. A possible explanation for the relatively large $q_{\text {surf }}$ at low density, is the following: at low source currents, radial electric fields cause a current to flow through the target, adding to the total dissipated heat [16]. Nevertheless, the power loss factor increases significantly with density, up to $f_{\text {pow }} \approx 25 \%$. We also note here that the total surface heat flux $q_{\text {surf }}$ includes neutral heating. The actual plasma power loss factor is larger than $f_{\text {pow }}$ calculated here.

The directly observed convection losses between $d=25$ and $3 \mathrm{~mm}$ amount to $10-20 \%$, without a significant correlation with density up to $n_{e}=8 \cdot 10^{20} \mathrm{~m}^{-3}$. It is noted that the power losses occur mostly within $3 \mathrm{~mm}$ from the target surface, and can only partly be probed by direct plasma measurements.

Momentum flux The total plasma pressure shows a linear dependence on density, with a slope of $\sim 60 \mathrm{~Pa} / 10^{20} \mathrm{~m}^{-3}$ (figure 6.b). This is reasonable, since static pressure $n_{e} e\left(T_{e}+T_{i}\right)$ is dominant at low $M$. A direct momentum loss of $10-30 \%$ is observed between $d=25$ and $3 \mathrm{~mm}$, with no significant correlation to $n_{e}$. The total momentum loss factor is estimated using equation 11, which can be solved for $f_{m o m}$ if $\Gamma_{s e}, p_{u}^{t o t}$, and $T_{s e}$ are known. Since the near-surface particle flux was found to be conserved in steady state operation, we set $\Gamma_{s e}=\Gamma_{u}$. Figure $6 . \mathrm{b}$ shows the direct measurements of $p_{u}^{\text {tot }}$, but $T_{s e}$ is difficult to obtain experimentally. We use the closest alternative instead: $T_{e, 3 \mathrm{~mm}}$. Given the consistent cooling towards the target, we expect $T_{e, 3 m m}>T_{s e}$. This estimation thus provides a lower bound for $f_{m o m}$. The results are shown in figure 6 .e, and at $n_{e}=8 \cdot 10^{20} \mathrm{~m}^{-3}$, provide a lower bound $f_{m o m} \geq 50 \%$.

\section{Processes of p-n coupling and estimation of flux loss factors}

In the previous section, it was shown that near-surface p-n coupling is present in Magnum-PSI, and its effects were assessed. We now consider the physical processes that drive this phenomenon. These include plasma-neutral interactions (discussed in section 5.1), but also the interaction with the wall itself (section 5.2).

\subsection{Interaction mean free paths}

Three p-n interactions are considered: recombination, ionization and Charge Exchange (CX), with corresponding collision rates from the AMJUEL database [34], equations 2.1.8, 2.2.9, and 3.1 .8 , respectively. The mean free path for these collisions gives an indication of their relevant length scale and, compared to the dimensions of the plasma and interaction region, whether they can significantly affect the near-surface plasma. is given by $\lambda_{m f p}=\frac{v_{r e l}}{\left\langle\sigma v_{r e l}\right\rangle n_{e}}$, with $\left\langle\sigma v_{r e l}\right\rangle$ the collision rate and $v_{r e l}$ the relative velocity between the colliding particles. The ionization and recombination rates depend on $n_{e}$ and $T_{e}$, and $v_{r e l}=v_{p}$ was used, representing the typical flow velocity of a moving ion or neutral particle. The CX rate is a function of $T_{e}$ and relative kinetic energy, therefore $v_{r e l}=c_{s}$ was used in this case, representing the opposite velocities of ions and neutrals. Using near-surface TS and CTS measurements of $n_{e}, T_{e}, T_{i}$, and $v_{p}$ as inputs, the mean free paths $\lambda_{m f p}$ for these collisions were estimated for each device setting in this study. 
The results are plotted as a function of $n_{e}$ in figure 7 .

The span of plasma radii $r_{p}$ during these experiments is also shown, for comparison. For

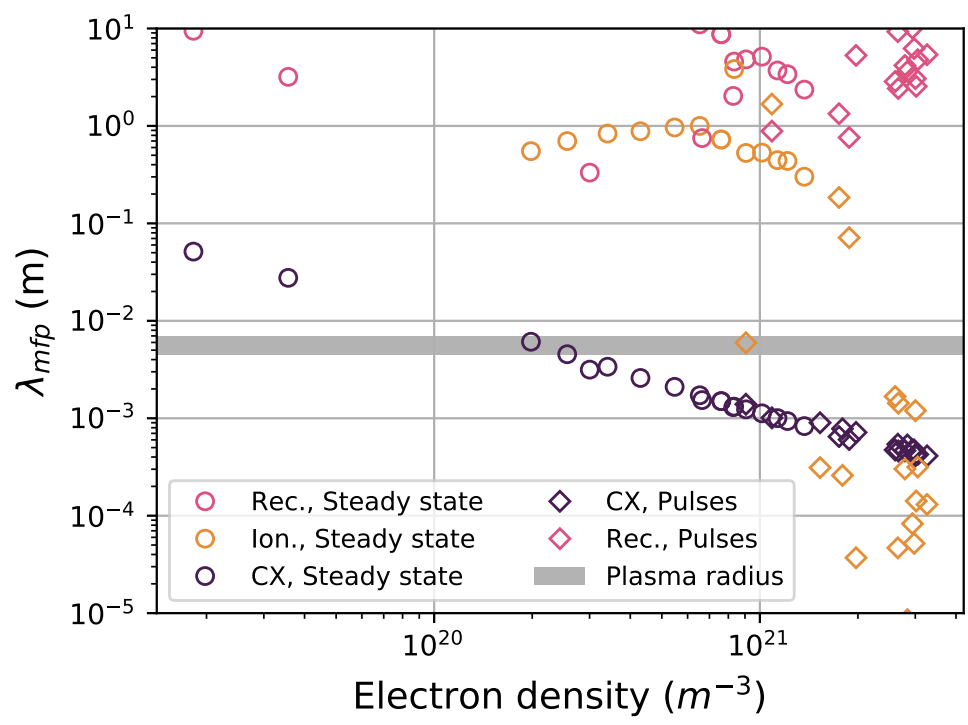

Figure 7. Mean free paths for CX, ionization and recombination as a function of plasma density, for the device settings described in section 2.3. For ionization and recombination, the flow velocity was assumed to equal the parallel plasma velocity, while for CX, the velocity of sound was used as relative velocity.

$\lambda_{m f p}<r_{p}$, the interaction can be expected to be present in the near-surface region. The estimations in figure 7 are in good agreement with the TS and CTS measurements discussed in section 4.1. At low densities, $\lambda_{m f p} \gg r_{p}$ for all reactions, the plasma is transparent to neutrals. These points correspond to the 8 and $10 P a$ cases shown in figure 5 , where electron cooling was indeed absent and pre-sheath acceleration was observed. On the other end of the domain are the plasma pulses. With $T_{e}=8-15 \mathrm{eV}$ and $n_{e}$ up to $3 \cdot 10^{21} \mathrm{~m}^{-3}$, these plasmas exhibit high rate coefficients for ionization as well as CX. The corresponding mean free paths are orders of magnitude below $r_{p}$, especially for ionization. Indeed, the upstream plasma is almost completely stagnated during these pulses, which indicates strong near-surface p-n coupling. In steady state exposure, $T_{e}$ and $n_{e}$ are lower, and the mean free paths for recombination as well as ionization are much larger than $r_{p}$. For CX, however, $\lambda_{m f p, C X}<r_{p}$ for $n_{e}>2 \cdot 10^{20} m^{-3}$ and scales inversely with $n_{e}$. This corresponds well to the negative correlation of $M_{u}$ with $n_{e}$ as reported in figure 3: the lower $\lambda_{m f p}$, the higher the total momentum loss per surface-recombined neutral particle. Therefore, CX is marked the dominant near-surface p-n interaction process in steady state operation of Magnum-PSI.

\subsection{Neutral flux amplification}

Next to volumetric interaction, the PFC surface itself also plays a role in the near-surface coupling of plasma and neutral parameters. To illustrate this, we follow a surface-recombined neutral on its way out of the plasma.

For $\lambda_{m f p, C X}<r_{p}$, the average neutral will exchange its charge, momentum and energy with a plasma particle before leaving the interaction zone. The corresponding plasma particle is neutralized. Dissipation of its energy commences through radiation, cross-field diffusion, or at the 
PFC surface. The latter route leads to a cycle of p-n interaction followed by surface relaxation. A particle can go through a number of interaction cycles before leaving the interaction region, thereby multiplying its contribution to $\mathrm{p}-\mathrm{n}$ coupling with a factor $K$, previously described as the neutral flux amplification factor [5, 35], and also described in [33]. The average value of $K$ depends on the near-surface system as a whole, with the main parameters being $T_{e}, n_{e}$, and the residence time of outwards diffusion surface-recombined neutrals. This process strongly increases the near-surface neutral density and interaction intensity, and is therefore proposed as the main driver of near-surface p-n coupling in Magnum-PSI during steady state operation. These processes underline the results described in section 4: a large fraction of the plasma momentum and energy flux is diverted and dissipated through neutrals, instead of flowing into the plasma sheath.

\section{Discussion and implications for ITER}

Although no direct measurements of neutral parameters were performed, the plasma flux measurements presented in this work demonstrate the presence of $\mathrm{p}-\mathrm{n}$ coupling in the neartarget region of Magnum-PSI. The effects of p-n coupling were observed for $n_{e}>2 \cdot 10^{20} \mathrm{~m}^{-3}$. A comparison of interaction mean free paths and the plasma radius, in section 5.1, indicates a similar density domain for $\mathrm{p}$ - $\mathrm{n}$ coupling to be present. Plasma-surface interaction is thus governed by the thermalized collisional pre-sheath in the marority of Magnum-PSI's operation domain, and expectedly in other dense thermal plasmas as well. The primary result is that in these conditions, a significant fraction of incident power is dissipated through neutrals, greatly reducing the sheath-transmitted PFC loads.

The impact on PFC loads is significant, and amounts to $f_{m o m} \geq 50 \%$ and $f_{\text {pow }} \geq 20 \%$ (still including surface heating by neutrals) for $n_{e} \geq 8 \cdot 10^{20} \mathrm{~m}^{-3}$. The directly measured momentum loss factors down to $d=3 \mathrm{~mm}$ are smaller than those from equation 11. Possibly, a significant fraction of p-n losses occur within $3 \mathrm{~mm}$ from the surface. This is especially plausible at high density: for $n_{e} \geq 10^{21} \mathrm{~m}^{-3}, \lambda_{C X} \leq 1 \mathrm{~mm}$. P-n coupling directly affects the PFC loads, but has other consequences as well. We mention three:

(i) Equilibration with the upstream plasma. Near-surface p-n coupling is very localized, but also affects the plasma flow upstream, and thus the SOL (and tokamak) plasma as a whole. In tokamak divertors, it is therefore important to assess its possible contributions to the onset of divertor detachment.

(ii) Power transfer to neutrals increases the neutral pressure and neutral energy dissipation, by diffusion or radiation. The PFC heat load is thus not only reduced in magnitude, but also shifted from sheath-accelerated ion bombardment and plasma electron heating to gaseous conduction.

(iii) Near-surface plasma cooling reduces $T_{s e}$. This leads to a lower Debye sheath potential, and reduces the ion impact energy and sputtering yields.

\subsection{Comparison to ITER conditions}

After reading that near-surface p-n coupling is relevant to PFC loads in Magnum-PSI, one might wonder how this translates to other devices. We have indicated three parameters of main importance to the presence of p-n coupling: $T_{e}, n_{e}$, and the residence time of surface-recombined neutrals in the interaction region. This work has shown that density is a factor of major importance: effects of $\mathrm{p}-\mathrm{n}$ coupling are present and increasing in intensity for $n_{e} \geq 2 \cdot 10^{20} \mathrm{~m}^{-3}$, but not observed for $n_{e} \leq 4 \cdot 10^{19} \mathrm{~m}^{-3}$. Therefore, $\mathrm{p}$-n coupling effects are not expected to be very significant in contemporary divertor tokamaks, where the density is usually in the order of magnitude $10^{19} \mathrm{~m}^{-3}$. In the ITER divertor, densities in the $10^{21} \mathrm{~m}^{-3}$ range are expected during high-recycling and detached operation scenarios [2] and therefore, some form of near-target p-n 
coupling is expected. The ITER divertor conditions differ from Magnum-PSI generally in three ways:

Firstly, higher plasma temperature: ranging from a few to a few tens of $e V$ between the (partially) detached and attached operation scenario's. In these conditions, near-surface ionization becomes dominant. The presented measurements during plasma pulses, performed at $T_{e}=8-15 \mathrm{eV}$, show an almost complete stagnation: $M<0.1$ (fig. 3), implying strong momentum losses. Based on the overlap in plasma temperatures between these experiments and the ITER divertor conditions, strong momentum losses are possibly present in ITER as well.

Secondly, lower plasma flow velocity. Magnum-PSI is a high-flux plasma generator with fluxes ca. $5-20$ times those in ITER (inferred from [2, fig. 6]). This is realised by a relatively large plasma flow velocity, i.e. Magnum-PSI is operated in a convective transport regime. The ITER divertor plasma, on the other hand, is mostly conductive, with a particle balance sustained by SOL ionization. The particle fluxes in ITER are lower than in Magnum-PSI, as is the surface recombination neutral source. However, p-n coupling and the corresponding flux losses depend on the amount of interaction per incident particle. A lower plasma velocity increases the ion transit time of the interaction region, and if anything, this is expected to increase the interaction rates and corresponding losses.

Thirdly, different system geometry. These experiments were performed with perpendicular incidence, while the ITER divertor operates under shallow incidence. The angle of incidence has been found to play an important role to p-n interaction in the divertor region [3]. Under perpendicular incidence, surface-recombined neutrals are generally reflected back into the plasma, while under shallow incidence, they are diverted away. Depending on the energy of reflected neutrals, the deflection angle reduces the residence time of surface-recombined neutrals, possibly resulting in lower near-surface losses.

In summary, the differences mentioned above do not exclude nor prescribe the presence of nearsurface p-n coupling in the ITER divertor. Working out the expected effects of these differences is a topic of future study. Nevertheless, it is possible that p-n coupling will be present in ITER. Given its significant impact on plasma-surface interaction, its effects should be adequately addressed in future PSI and SOL studies.

\subsection{Integration of near-target $p$-n coupling in SOL models}

The near-target plasma is a multi-faceted system; it is an equilibrium of many properties, processes and interactions that determines the loss factors $f_{\text {mom }}$ and $f_{\text {pow }}$. Current finite-element SOL models are well developed [36] and in principle able to describe the near-surface system as part of the global SOL or linear plasma beam. These models contain the relevant physics and collision data, which includes not only atomic collisions, but also molecular effects, kinetic neutrals and the angular and energetic distributions of reflected particles. These processes and properties are part of the near-surface system and affect the resulting loss factors. In principle, SOL models are thus well-suited to include near-surface p-n coupling implicitly. However, p$\mathrm{n}$ coupling processes are very localized, and result in strong gradients of plasma and neutral parameters near the surface. In particular, many relevant reaction rates depend strongly on $T_{e}$, and $T_{e}$ is strongly affected by p-n coupling as well. Therefore, $T_{e}$ is a key parameter in this system, and should be properly resolved in finite-element SOL models. We also note that the boundary value, $T_{s, s e}$, does not only play a key role in the Debye sheath, but is also regarded an important parameter to SOL physics in general, as stated in references [35, 7], and proposed as an indicator for divertor detachment [5]. This parameter is at risk of being ill-defined in finite-element models with a coarse grid near the target. To properly resolve p-n coupling, the near-target parallel grid size, $d_{\|}$, should adhere

$$
d_{\|} \leq \lambda_{m f p},
$$


with $\lambda_{m f p}$ the neutral mean free path for p-n interaction in general, including elastic as well as inelastic collisions. For Magnum-PSI in steady state operation, CX is the limiting factor, which sets the requirement $d_{\|} \leq \lambda_{C X} \sim 1 \mathrm{~mm}$. In ITER, ionization will be dominant, which potentially brings $d_{\|}$below $10^{-4} \mathrm{~m}$.

For very short collision length scales, two issues arise. Firstly, for $\lambda_{m f p}$ smaller than the Debye sheath dimensions, the sheath becomes collisional, and the SOL model boundary condition has to be fundamentally adapted. Under shallow incidence, collisionality in the magnetic pre-sheath should also be accounted for. Secondly, a fine grid comes with a computational cost. This issue can be averted by including p-n coupling explicitly. For example, one could set up a global model that estimates flux loss factors as a function of near-surface plasma parameters. This model could serve as a boundary condition to the SOL model, and can also be used to compute PFC loads, through the sheath as well as neutral dissipation. Such a model can be formulated analytically, which inherently requires certain simplifications, but could possibly results in a flexible tool. Alternatively, a p-n coupling model can be obtained through parametrization of dedicated finite element or particle-in-cell modeling of the near-surface region.

In conclusion, this study confirms that near-target p-n coupling is present in Magnum-PSI, and facilitates substantial momentum and energy losses within a few $\mathrm{mm}$ from the target surface. In this thermalized collisional pre-sheath, substantial amounts of pressure and energy are diverted from the sheath-transmitted plasma flux, into neutral gas. These processes affect PFC loads as well as the upstream plasma flow, and can possibly play a role in the onset of divertor detachment in present or future fusion devices.

Acknowledgements The DIFFER co-workers and especially the Magnum-PSI Facility Team are warmly thanked for their practical help and insights. The Magnum-PSI facility at DIFFER has been funded by the Netherlands Organisation for Scientific Research (NWO) and EURATOM. This work has been carried out within the framework of the EUROfusion Consortium and has received funding from the Euratom research and training programme 20142018 and 2019-2020 under grant agreement No 633053. The views and opinions expressed herein do not necessarily reflect those of the European Commission.

\section{References}

[1] P.C. Stangeby. The plasma boundary of magnetic fusion devices. Plasma physics series. Institute of Physics Pub, Bristol ; Philadelphia, 2000. ISBN 978-0-7503-0559-4.

[2] R.A. Pitts, X. Bonnin, F. Escourbiac, H. Frerichs, J.P. Gunn, T. Hirai, A.S. Kukushkin, E. Kaveeva, M.A. Miller, D. Moulton, V. Rozhansky, I. Senichenkov, E. Sytova, O. Schmitz, P.C. Stangeby, G. De Temmerman, I. Veselova, and S. Wiesen. Physics basis for the first ITER tungsten divertor. Nuclear Materials and Energy, 20:100696, August 2019. ISSN 23521791. doi: 10.1016/j.nme.2019.100696. URL https://linkinghub.elsevier.com/ retrieve/pii/S2352179119300237.

[3] A. Fil, B. Lipschultz, D. Moulton, B.D. Dudson, O. Février, O. Myatra, C. Theiler, K. Verhaegh, M. Wensing, EUROfusion MST1 teams, and the TCV team. Separating the roles of magnetic topology and neutral trapping in modifying the detachment threshold for TCV. Plasma Physics and Controlled Fusion, 62(3):035008, March 2020. ISSN 07413335, 1361-6587. doi: 10.1088/1361-6587/ab69bb. URL https://iopscience.iop.org/ article/10.1088/1361-6587/ab69bb.

[4] G.R.A. Akkermans, I.G.J. Classen, H.J. van der Meiden, J. van den Berg-Stolp, and J.W.M. Vernimmen. The role of hydrogen molecular effects on detachment in Magnum-PSI. Submitted., 2021. 
[5] P.C. Stangeby. Basic physical processes and reduced models for plasma detachment. Plasma Physics and Controlled Fusion, 60(4):044022, April 2018. ISSN 0741-3335, 13616587. doi: 10.1088/1361-6587/aaacf6. URL http://stacks.iop.org/0741-3335/60/i= 4/a=044022 ?key=crossref . 34a2c3a50b5467333f4072ed223e94b7.

[6] K. Verhaegh and et al. An improved understanding of the roles of atomic processes and power balance in divertor target ion current loss during detachment. Nuclear Fusion, 2019. doi: 10.1088/1741-4326/ab4251.

[7] P.C. Stangeby and C. Sang. Strong correlation between $D_{2}$ density and electron temperature at the target of divertors found in SOLPS analysis. Nuclear Fusion, 57(5): 056007, May 2017. ISSN 0029-5515, 1741-4326. doi: 10.1088/1741-4326/aa5e27. URL https://iopscience.iop.org/article/10.1088/1741-4326/aa5e27.

[8] A.E. Shumack. The influence of electric fields and neutral particles on the plasma sheath at ITER divertor conditions. $\mathrm{PhD}$ thesis, Technische Universiteit Eindhoven, 2011. URL https://research.tue.nl/en/publications/ the-influence-of-electric-fields-and-neutral-particles-on-the-plasma-sheath-at-iter-di .html.

[9] M.A. van den Berg, K. Bystrov, R. Pasquet, J.J. Zielinski, and G. De Temmerman. Thermographic determination of the sheath heat transmission coefficient in a high density plasma. Journal of Nuclear Materials, 438:S431-S434, July 2013. ISSN 00223115. doi: 10. 1016/j.jnucmat.2013.01.087. URL https://linkinghub.elsevier.com/retrieve/pii/ S0022311513000950.

[10] J. van den Berg, H.J. van der Meiden, J.W.M. Vernimmen, and I.G.J. Classen. Thomson scattering near the high-fluence target surface of the Magnum-PSI linear plasma generator. Journal of Instrumentation, 14(10):C10041-C10041, October 2019. ISSN 1748-0221. doi: 10.1088/1748-0221/14/10/C10041. URL https://iopscience.iop.org/article/ 10.1088/1748-0221/14/10/C10041.

[11] W.A.J. Vijvers, C.A.J. van Gils, W.J. Goedheer, H.J. van der Meiden, D.C. Schram, V.P. Veremiyenko, J. Westerhout, N.J. Lopes Cardozo, and G.J. van Rooij. Optimization of the output and efficiency of a high power cascaded arc hydrogen plasma source. Physics of Plasmas, 15(9):093507, September 2008. ISSN 1070-664X, 1089-7674. doi: 10.1063/1.2979703. URL http://aip.scitation.org/doi/10.1063/1.2979703.

[12] H.J.N. van Eck, G.R.A. Akkermans, S. Alonso van der Westen, D.U.B. Aussems, M. van Berkel, S. Brons, I.G.J. Classen, H.J. van der Meiden, T.W. Morgan, M.J. van de Pol, J. Scholten, J.W.M. Vernimmen, E.G.P. Vos, and M.R. de Baar. High-fluence and high-flux performance characteristics of the superconducting Magnum-PSI linear plasma facility. Fusion Engineering and Design, 142:26-32, May 2019. ISSN 09203796. doi: 10.1016/j.fusengdes.2019.04.020. URL https://linkinghub.elsevier.com/retrieve/ $\mathrm{pii/S092037961930554X.}$

[13] T.W. Morgan, M.A. van den Berg, G. De Temmerman, S. Bardin, D.U.B. Aussems, and R.A. Pitts. Power deposition on misaligned castellated tungsten blocks in the MagnumPSI and Pilot-PSI linear devices. Nuclear Fusion, 57(12):126025, December 2017. ISSN 0029-5515, 1741-4326. doi: 10.1088/1741-4326/aa8109. URL http://stacks.iop.org/ $0029-5515 / 57 / i=12 / a=126025$ ? key=crossref . bad717b35203e017718062dfff $203 \mathrm{e} 26$.

[14] T.W. Morgan, M. Balden, T. Schwarz-Selinger, Y. Li, T.H. Loewenhoff, M. Wirtz, S. Brezinsek, and G. de Temmerman. ITER monoblock performance under lifetime loading conditions in Magnum-PSI. Physica Scripta, T171:014065, January 2020. ISSN 00318949, 1402-4896. doi: 10.1088/1402-4896/ab66df. URL https://iopscience.iop.org/ article/10.1088/1402-4896/ab66df. 
[15] R. Perillo, G.R.A. Akkermans, I.G.J. Classen, W.A.J. Vijvers, R. Chandra, K. Jesko, S. Korving, J.W.M. Vernimmen, and M.R. de Baar. Experimental evidence of enhanced recombination of a hydrogen plasma induced by nitrogen seeding in linear device MagnumPSI. Nuclear Materials and Energy, 19:87-93, May 2019. ISSN 23521791. doi: 10.1016/j.nme.2019.02.018. URL https://linkinghub.elsevier.com/retrieve/pii/ S2352179118300838.

[16] G.R.A. Akkermans, I.G.J. Classen, R. Perillo, H.J. van der Meiden, F. Federici, and S. Brezinsek. The role of hydrogen molecular effects on detachment in Magnum-PSI. Physics of Plasmas, 27(10):102509, October 2020. ISSN 1070-664X, 1089-7674. doi: 10.1063/5.0017714. URL http://aip.scitation.org/doi/10.1063/5.0017714.

[17] G.G. van Eden, V. Kvon, M.C.M. van de Sanden, and T.W. Morgan. Oscillatory vapour shielding of liquid metal walls in nuclear fusion devices. Nature Communications, 8 (1), December 2017. ISSN 2041-1723. doi: 10.1038/s41467-017-00288-y. URL http: //www.nature.com/articles/s41467-017-00288-y.

[18] P. Rindt, T.W. Morgan, G.G. van Eden, M.A. Jaworski, and N.J. Lopes Cardozo. Power handling and vapor shielding of pre-filled lithium divertor targets in MagnumPSI. Nuclear Fusion, 59(5):056003, May 2019. ISSN 0029-5515, 1741-4326. doi: 10. 1088/1741-4326/ab0560. URL http://stacks.iop.org/0029-5515/59/i=5/a=056003? key=crossref . af31757f8f59c7ee05ea91f6a09ee0c7.

[19] H.J. van der Meiden, A.R. Lof, M.A. van den Berg, S. Brons, A.J.H. Donné, H.J.N. van Eck, P.M.J. Koelman, W.R. Koppers, O.G. Kruijt, N.N. Naumenko, T. Oyevaar, P.R. Prins, J. Rapp, J. Scholten, D.C. Schram, P.H.M. Smeets, G. van der Star, S.N. Tugarinov, and P.A. Zeijlmans van Emmichoven. Advanced Thomson scattering system for high-flux linear plasma generator. Review of Scientific Instruments, 83(12):123505, December 2012. ISSN 0034-6748, 1089-7623. doi: 10.1063/1.4768527. URL http: //aip.scitation.org/doi/10.1063/1.4768527.

[20] H.J. van der Meiden, J.W.M. Vernimmen, K. Bystrov, K. Jesko, M.Y. Kantor, G. De Temmerman, and T.W. Morgan. Collective Thomson scattering system for determination of ion properties in a high flux plasma beam. Applied Physics Letters, 109 (26):261102, December 2016. ISSN 0003-6951, 1077-3118. doi: 10.1063/1.4973211. URL http://aip.scitation.org/doi/10.1063/1.4973211.

[21] D.E. Evans and J. Katzenstein. Laser light scattering in laboratory plasmas. Reports on Progress in Physics, 32(1):207-271, January 1969. ISSN 00344885. doi: 10. 1088/0034-4885/32/1/305. URL http://stacks.iop.org/0034-4885/32/i=1/a=305? key=crossref . d8cb0d72bac877ac0c0690b2a16de9cc.

[22] H.J. van der Meiden. Thomson scattering on low and high temperature plasmas. PhD thesis, Technische Universiteit Eindhoven, 2011. URL http://repository.tue.nl/694404.

[23] I. Abramovic, A. Pavone, J. Svensson, D. Moseev, M. Salewski, H.P. Laqua, N.J. Lopes Cardozo, and R.C. Wolf. Collective Thomson scattering data analysis for Wendelstein 7X. Journal of Instrumentation, 12(08):C08015-C08015, August 2017. ISSN 1748-0221. doi: 10.1088/1748-0221/12/08/C08015. URL https://iopscience.iop.org/article/ 10.1088/1748-0221/12/08/C08015.

[24] D. Foreman-Mackey, D.W. Hogg, D. Lang, and J. Goodman. emcee : The MCMC Hammer. Publications of the Astronomical Society of the Pacific, 125(925):306-312, March 2013. ISSN 00046280, 15383873. doi: 10.1086/670067. URL http://iopscience.iop.org/article/ 10.1086/670067.

[25] I. H. Hutchinson. Principles of plasma diagnostics. Cambridge University Press, Cambridge ; New York, 2nd ed edition, 2002. ISBN 978-0-521-80389-2. 
[26] C. Costin, V. Anita, G. Popa, J. Scholten, and G. De Temmerman. Tailoring the charged particle fluxes across the target surface of magnum-psi. Plasma Sources Science and Technology, 25:025023, 2016. doi: 10.1088/0963-0252/25/2/ 025023. URL http://www. euro-fusionscipub.org/wp-content/uploads/eurofusion/ WPPFCPR15_14239_submitted.pdf.

[27] Y. Hayashi, K. Ješko, H.J. van der Meiden, J.W.M. Vernimmen, T.W. Morgan, N. Ohno, S. Kajita, M. Yoshikawa, and S. Masuzaki. Plasma detachment study of high density helium plasmas in the Pilot-PSI device. Nuclear Fusion, 56(12):126006, December 2016. ISSN 00295515, 1741-4326. doi: 10.1088/0029-5515/56/12/126006. URL http://stacks.iop.org/ 0029-5515/56/i=12/a=126006?key=crossref . 7 aedae56896787aa018b3c68b3659e21.

[28] J.P. Gunn, C. Boucher, B.L. Stansfield, and C.S. Maclatchy. Flush-mounted Probes in the TdeV Tokamak: Theory and Experiment. Contributions to Plasma Physics, 36(S1):45-52, 1996. ISSN 08631042. doi: 10.1002/ctpp.19960360108. URL http://doi.wiley.com/10. 1002/ctpp. 19960360108.

[29] W. Zhao, Y. Wang, Y. Jin, L. Zhao, H. Zhou, G. Nie, L.and Zhong, C. Liu, C. Watts, and J.P. Gunn. Preliminary Design of ITER Divertor Langmuir Probe System. Fusion Science and Technology, 76(2):79-87, February 2020. ISSN 1536-1055, 1943-7641. doi: 10. 1080/15361055.2019.1674123. URL https://www.tandfonline.com/doi/full/10.1080/ 15361055.2019 .1674123$.

[30] Y. Li, T.W. Morgan, J.A.W. Van Dommelen, S. Antusch, M. Rieth, J.P.M. Hoefnagels, D. Terentyev, G. De Temmerman, K. Verbeken, and M.G.D. Geers. Fracture behavior of tungsten-based composites exposed to steady-state/transient hydrogen plasma. Nuclear Fusion, 60(4):046029, April 2020. ISSN 0029-5515, 1741-4326. doi: 10.1088/1741-4326/ ab77e7. URL https://iopscience.iop.org/article/10.1088/1741-4326/ab77e7.

[31] T.W. Morgan, T.M de Kruif, H.J. van der Meiden, M.A. van den Berg, J. Scholten, W. Melissen, B.J.M. Krijger, S. Bardin, and G. de Temmerman. A high-repetition rate edge localised mode replication system for the Magnum-PSI and Pilot-PSI linear devices. Plasma Physics and Controlled Fusion, 56(9):095004, September 2014. ISSN 0741-3335, 1361-6587. doi: 10.1088/0741-3335/56/9/095004. URL https://iopscience.iop.org/ article/10.1088/0741-3335/56/9/095004.

[32] S. Kuhn, K.U. Riemann, N. Jelić, D.D. Tskhakaya, D. Tskhakaya, and M. Stanojević. Link between fluid and kinetic parameters near the plasma boundary. Physics of Plasmas, 13 (1):013503, January 2006. ISSN 1070-664X, 1089-7674. doi: 10.1063/1.2161181. URL http://aip.scitation.org/doi/10.1063/1.2161181.

[33] P.C. Stangeby. Can detached divertor plasmas be explained as self-sustained gas targets? Nuclear Fusion, 33(11):1695-1705, November 1993. ISSN 0029-5515. doi: 10.1088/0029-5515/33/11/I10. URL https://iopscience.iop.org/article/10.1088/ 0029-5515/33/11/I10.

[34] D. Reiter. The data file amjuel: Additional atomic and molecular data for eirene. 012000.

[35] V. Kotov and D. Reiter. Two-point analysis of the numerical modelling of detached divertor plasmas. Plasma Physics and Controlled Fusion, 51(11):115002, November 2009. ISSN 07413335, 1361-6587. doi: 10.1088/0741-3335/51/11/115002. URL http://stacks.iop.org/ 0741-3335/51/i=11/a=115002?key=crossref . 34bafe28de26874ec2dd98bf5334a265.

[36] S. Wiesen, D. Reiter, V. Kotov, M. Baelmans, W. Dekeyser, A.S. Kukushkin, S.W. Lisgo, R.A. Pitts, V. Rozhansky, G. Saibene, I. Veselova, and S. Voskoboynikov. The new SOLPSITER code package. Journal of Nuclear Materials, 463:480-484, August 2015. ISSN 00223115. doi: 10.1016/j.jnucmat.2014.10.012. URL https://linkinghub.elsevier.com/ retrieve/pii/S0022311514006965. 